Historia y comunicación social

ISSN: $1137-0734$

http://dx.doi.org/10.5209/hics.65124

\title{
Creencias acerca de los mitos del amor romántico entre el alumnado de Comunicación Audiovisual de la Universidad Complutense de Madrid
}

\author{
María Goenaga Ruiz de Zuazu'
}

Recibido el: 16 de julio de 2019 / Aceptado: 2 de julio de 2020

Resumen. Asumiendo la importancia de romper con los estereotipos del amor romántico, y siendo el mundo audiovisual donde estos ideales están muy presentes, analizamos si alumnos/as del Grado Comunicación Audiovisual perpetúan o no estos mitos. Aplicamos un cuestionario para evaluar las creencias amorosas y la justificación de la violencia de género a una muestra de 149 alumnos/as del Grado de CAV de la UCM. Nuestros resultados muestran un claro rechazo a la violencia de género y, en general, no se perpetúan los mitos del amor romántico entre el alumnado. Sin embargo, variables como el tipo de colegio, la familia o la edad del alumno/a pueden estar influyendo negativamente en el mantenimiento de algunos mitos en torno principalmente a la omnipotencia o la perdurabilidad del amor.

Palabras clave: Amor romántico; universidad; socialización; cine; mitos; relatos audiovisuales; España.

\section{[en] Beliefs about the myths of romantic love among Audiovisual Communication students at UCM}

\begin{abstract}
Understanding the importance of breaking away from stereotypes surrounding romantic love, and because such ideals are so strongly present in the audiovisual world, we examine whether students studying a Degree in Audiovisual Communication are perpetuating these myths or not. We administered a questionnaire to evaluate romantic beliefs and the justification of gender violence to a sample of 149 students enrolled on the Degree in Audiovisual Communication taught at UCM. Our results show a clear rejection of gender violence and that, in general, the myths of romantic love are not being perpetuated among the students. However, variables such as the type of school attended, family, or age of the student could negatively influence the continuance of certain myths, principally with regard to the omnipotence or durability of love.
\end{abstract}

Keywords: Romantic love; university; socialization; cinema; myths; audiovisual stories; Spain.

Sumario: 1. Introducción. 2. Objetivos e hipótesis. 3. Metodología. 3.1. Muestra. 3.2. Instrumentos: escalas utilizadas. 3.3. Análisis de datos. 4. Resultados. 4.1. Mitos del amor romántico. 4.2. Justificación de la violencia de género. 5. Conclusiones y discusión. 6. Referencias bibliogfáficas. 7. Anexos. 7.1. Anexo 1: cuestionario.

Cómo citar: Goenaga Ruiz de Zuazu, M. (2020) Creencias acerca de los mitos del amor romántico entre el alumnado de Comunicación Audiovisual de la Universidad Complutense de Madrid, Historia y comunicación social 26(1), 263-277.

\section{Introducción}

Cada período histórico ha desarrollado una concepción diferente del amor y, en consecuencia, los elementos en torno al sexo o al matrimonio han ido cambiando a lo largo del tiempo (Barrón et al., 1999; Yela, 2000, 2003).

El amor romántico, tal y como es entendido hoy en España, es una construcción socio-cultural de Occidente (Aron, Fisher y Mashek, 2005; Chan y Xueli, 2011; Neves, 2007; Stone, 1989) heredada del amor cortés, burgués y victoriano. Se trata de una construcción idealizada en torno a unos mitos (resumidos en la tabla 1) considerados como un conjunto de creencias socialmente compartidas sobre la "supuesta verdadera naturaleza del amor" (Yela, 2003: 264). Cuando esta construcción "falla", genera frustración e insatisfacción por no llegar a cumplir las expectativas (Yela, 2003: 264); distorsiona la capacidad de los individuos para resolver las dificultades que surgen cuando falta el amor (Bradbury y Fincham, 1990: 8); pero sobre todo, dificulta el establecimiento de relaciones sanas y la tolerancia a comportamientos abusivos (Fundación Mujeres, 2011: 3).

\footnotetext{
Universidad Complutense de Madrid

E-mail:mgoenaga@ucm.es

ORCID: https://orcid.org/0000-0002-2276-770X
}

Hist. comun. soc. 26(1) 2021: 263-277 
Tabla 1. Mitos del amor romántico

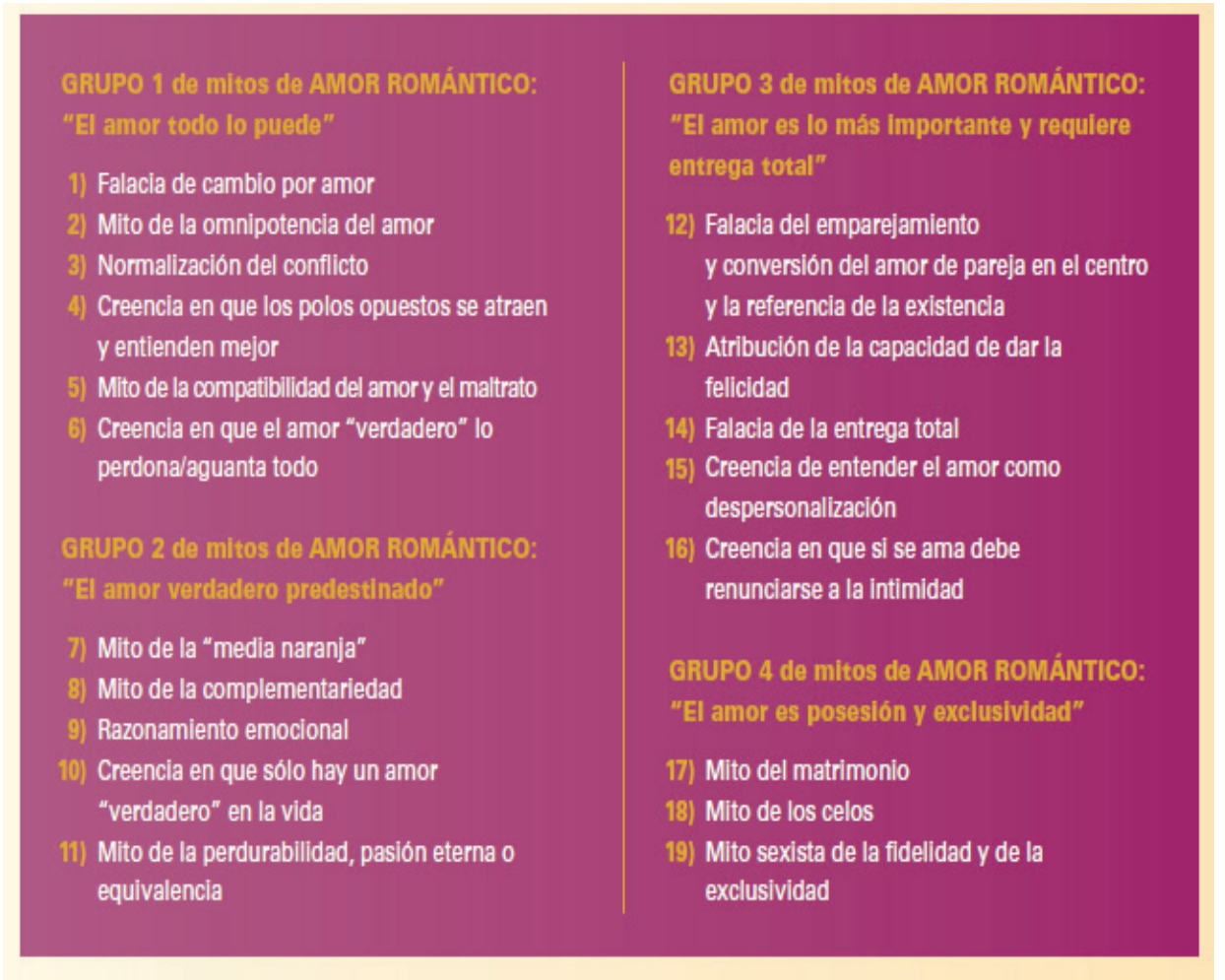

Fuente: Fundación Mujeres (2011: 7).

A pesar de que estos mitos son ficticios, engañosos e irracionales, suelen mantenerse en el tiempo por su transmisión a través del proceso de socialización ${ }^{2}$. Es mediante este proceso cuando los individuos se conforman una identidad género, aprendiendo los roles, normas o estereotipos que dicta su cultura. En este proceso, conocido como teoría de la socialización diferencial, es donde se impulsa la socialización de género y la construcción social del amor asentado en los mitos de los que hemos hablado. Y para ello, los agentes de socialización (familia, escuela, medios de comunicación, etc.) son quienes juegan un papel clave en perpetuar o no estos estereotipos. Desde una perspectiva de género, se critican estos mitos (Ferrer et al., 2010: 393) por reforzar el papel pasivo y subordinado de la mujer frente al hombre en una sociedad patriarcal.

En concreto, son los medios de comunicación los agentes socializadores más importantes entre la juventud puesto que son además, grupos de referencia ${ }^{3}$ para construir su identidad (Falcón y Díaz-Aguado, 2014: 153). Son muchos los estudios que han demostrado cómo a través de la pantalla se puede aprender cómo deben ser las relaciones amorosas, cultivándose creencias en la audiencia en torno a los mitos del amor romántico (Ferris et al., 2007; Lee, 2008; Nabi et al., 2006; Winn, 2007).

Pero, lo más preocupante hoy en día, es que estos mitos no sólo están presentes en películas "Disney" (donde la ficción es muy explícita) sino que cada vez están presentes en muchas películas y series (Chan y Xueli, 2011; Hefner y Wilson, 2013), donde los modelos han pasado de príncipes y princesas a vampiros y vampiresas (por ejemplo, en "Crepúsculo"). De hecho, estudios recientes en nuestro país (Marmol-Martín et al., 2018: 74) confirman que la juventud expone como referentes de sus ideales amorosos a personajes de estos productos audiovisuales. Menéndez (2014: 31) afirma que todavía es mayoritaria una cinematografía que obedece a problemas sexistas y que reproduce diversos tipos de violencia, como la sexual o la simbólica. De hecho, en el estudio de Bernárdez et al. (2008: 122) donde se analizan las películas más taquilleras del cine español, encuentran estereotipos sexistas en todas ellas.

Aunque, como han confirmado varios estudios (Duque, 2010-2011; Yuste et al., 2014), los roles tradiciones de género no equivalen directamente a maltrato y violencia de género, indudablemente generan una visión sexista de las relaciones amorosas que trasciende de la pantalla a la realidad. Además, es importante subrayar que investigaciones realizadas en España (Díaz-Aguado et al, 2013) reflejan cómo los/las adolescentes entienden los medios audiovisuales como su principal fuente de información sobre cómo entender la violencia de género; y siguen manteniendo el mito de que "los celos son una expresión de amor" (36\%), lo cual está incrementando el riesgo de violencia de género de esta generación.

Proceso mediante el cual las personas aprendemos, construimos e interiorizamos los valores, creencias, actitudes, expectativas y comportamientos característicos de la sociedad en la que nacemos (Giddens, 2001).

Un grupo ajeno al que nosotros/as pertenecemos pero que, sin embargo, ordena nuestro comportamiento (Hyman, 1942). 
Son varios los estudios con población adulta o concretamente universitaria (e incluso con adolescentes), como el nuestro, que confirman la diferente concepción del amor entre hombres y mujeres (Carreño y Serrano, 1995; Díaz-Aguado y Carvajal, 2012; Esteban y Távora, 2008; Ferrer, Bosch y Navarro, 2010; Moreno et al., 2007; Rodríguez-Castro et al., 2013). Mientras en gran medida las mujeres creen en el mito de la media naranja, la perdurabilidad y la omnipotencia del amor; los hombres dan más importancia a la pasión sexual y a los celos como prueba amorosa.

Además, cabe destacar cómo existe un amplio reconocimiento del papel de la Universidad en la construcción de la igualdad y la prevención de violencia contra las mujeres (Díaz-Aguado y Carvajal, 2012; FernándezEnguita, 2014; Tajahuerce y Ramírez (Eds.), 2018).

Por todo lo anterior, parece pertinente un estudio como éste en donde la muestra es el alumnado del Grado de Comunicación Audiovisual (en adelante, CAV) por su importante papel como futuros agentes de socialización de género en el mundo de los relatos audiovisuales. Serán ellos/as en un futuro cercano quienes, delante o detrás de las cámaras, diseñen las relaciones amorosas. Habiéndose encontrado evidencias empíricas entre la juventud de una perpetuación de los estereotipos de género, parece imprescindible estudiar si este perfil de alumnado universitario mantiene o no los mitos del amor romántico y, a su vez, justifica la violencia de género. Su papel será clave para deconstruir los estereotipos de género en torno al amor, por lo que es pertinente analizar cómo interpretan estas cuestiones.

\section{Objetivos e hipótesis}

Aunque encontramos investigaciones recientes en nuestro país (Bonilla-Algovia y Rivas-Rivero, 2018; Caro y Monreal, 2017; Fernández y Navas, 2016; Ferrer, Bosch y Navarro, 2010; García-Cueto et al., 2015; RodríguezCastro et al., 2013; Rodríguez-Santero et al., 2017) donde se estudian las características de los mitos del amor romántico o la justificación de la violencia de género entre adolescentes o universitarios/as, no se han realizado estudios centrados en un perfil de alumnado de CAV como agentes de socialización en torno al amor.

En este sentido, nuestro objetivo general es: "Comprobar si persiste algún mito de amor romántico entre el alumnado de CAV de la UCM". Y como objetivos específicos nos proponemos los siguientes:

- Analizar qué mitos del amor romántico persisten entre este perfil de alumnado universitario.

- Estudiar si la variable "socialización primaria" (familia y escuela) provoca diferencias en la asunción de los ideales del amor romántico en este perfil de estudiantes.

- Estudiar si la variable "socialización primaria" (familia y escuela) provoca diferencias en la justificación de la violencia de género en este perfil de estudiantes.

- Identificar si existen diferencias de género entre los ideales del amor romántico entre el alumnado de CAV.

- Identificar si existen diferencias de género en la justificación de la violencia de género entre el alumnado de CAV.

En cuanto a nuestras hipótesis de estudio, trataremos de confirmar o refutar las siguientes:

- H1: El alumnado universitario de CAV no justifica la violencia de género, independientemente de su sexo.

- H2: Encontramos diferencias de género en la interpretación de los mitos del amor romántico.

- H3: Es el alumnado más joven quien en mayor medida perpetúa algunos mitos del amor romántico.

- H4: El tipo de centro educativo en el que ha estudiado el alumnado universitario de CAV provoca diferentes interpretaciones del amor romántico.

\section{Metodología}

\subsection{Muestra}

Para cumplir con nuestros objetivos, realizamos una investigación cuantitativa basada en encuesta. El tipo de muestreo usado fue de tipo no probabilístico accidental, común en este tipo de investigaciones (Grande y Abascal 2005; McMillan y Schumacher 2005; Rodriguez-Santero et al., 2017), enviándose a través de "formularios de Google" el cuestionario anonimizado a un grupo de alumnos/as y varios de exalumnos/as de la asignatura de "Sociología" durante el segundo semestre del curso 2018-19 (entre los meses de marzo y mayo). La participación en la encuesta era voluntaria y se ha obtenido una muestra total de 149 alumnos/as. Si bien nuestro muestreo no nos permite extrapolar los resultados al conjunto del alumnado de CAV ni mucho 
menos a todo el ámbito universitario (validez externa), sí nos permite tener una aproximación del problema en este perfil de alumnado y más habiendo obtenido participación de todos los cursos del Grado. La siguiente tabla (tabla 2) resume los datos sociodemográficos de los participantes en el estudio:

Tabla 2. Perfil sociodemográfico de la muestra

\begin{tabular}{|c|c|c|c|}
\hline VARIABLES & CATEGORIAS & ABSOLUTOS & PORCENTAJES \\
\hline Curso & $\begin{array}{l}\text { Primero } \\
\text { Segundo } \\
\text { Tercero } \\
\text { Cuarto }\end{array}$ & $\begin{array}{l}67 \\
32 \\
18 \\
32\end{array}$ & $\begin{array}{l}45,0 \% \\
21,5 \% \\
12,0 \% \\
21,5 \%\end{array}$ \\
\hline Edad & \begin{tabular}{|l}
18 años \\
19 años \\
20 años \\
21 años \\
Más de 21
\end{tabular} & $\begin{array}{l}37 \\
39 \\
22 \\
25 \\
26 \\
\end{array}$ & $\begin{array}{l}24,9 \% \\
26,2 \% \\
14,8 \% \\
16,8 \% \\
17,5 \%\end{array}$ \\
\hline Sexo & $\begin{array}{l}\text { Hombre } \\
\text { Mujer }\end{array}$ & $\begin{array}{c}48 \\
101\end{array}$ & $\begin{array}{l}32,2 \% \\
67,8 \%\end{array}$ \\
\hline Pareja & $\begin{array}{l}\text { Sí } \\
\text { No }\end{array}$ & $\begin{array}{l}55 \\
94\end{array}$ & $\begin{array}{l}36,9 \% \\
63,1 \%\end{array}$ \\
\hline Tipo de centro educativo & \begin{tabular}{|l} 
Público \\
Privado o concertado laico \\
Privado o concertado religioso \\
\end{tabular} & $\begin{array}{l}90 \\
35 \\
24\end{array}$ & $\begin{array}{l}60,4 \% \\
23,5 \% \\
16,1 \% \\
\end{array}$ \\
\hline Nivel estudios padre & $\begin{array}{l}\text { No terminó la primaria } \\
\text { Estudios primarios } \\
\text { Estudios secundarios } \\
\text { Diplomatura o carrera media } \\
\text { Licenciatura universitaria } \\
\text { Doctorado o Máster }\end{array}$ & $\begin{array}{c}2 \\
22 \\
51 \\
20 \\
39 \\
15\end{array}$ & $\begin{array}{l}1,3 \% \\
14,8 \% \\
34,2 \% \\
13,4 \% \\
26,2 \% \\
10,1 \% \\
\end{array}$ \\
\hline Nivel estudios madre & $\begin{array}{l}\text { No terminó la primaria } \\
\text { Estudios primarios } \\
\text { Estudios secundarios } \\
\text { Diplomatura o carrera media } \\
\text { Licenciatura universitaria } \\
\text { Doctorado o Máster }\end{array}$ & $\begin{array}{c}3 \\
16 \\
57 \\
10 \\
46 \\
17\end{array}$ & $\begin{array}{c}2,0 \% \\
10,7 \% \\
38,3 \% \\
6,7 \% \\
30,9 \% \\
11,4 \%\end{array}$ \\
\hline Estilo educativo familiar & $\begin{array}{l}\text { Autoritario } \\
\text { Permisivo } \\
\text { Democrático }\end{array}$ & $\begin{array}{c}9 \\
47 \\
93\end{array}$ & $\begin{array}{c}6,0 \% \\
31,5 \% \\
62,4 \%\end{array}$ \\
\hline
\end{tabular}

Fuente: elaboración propia

\subsection{INSTRUMENTOS: ESCALAS UTILIZADAS}

En primer lugar, y con el objetivo de evaluar los "mitos del amor romántico", usamos como instrumento de medida la escala "Romantic Beliefs Scale (ROMBEL)" desarrollada por Sprecher y Metts (1989). Se trata de la escala más empleada internacionalmente para evaluar las creencias románticas por su amplia fiabilidad (alfa de Cronbach= 0.81) pero, además, y como demuestran estudios posteriores, cuenta con coexistencia interna (Jones y Cunningham, 1996; Sharp y Ganong, 2000) y altos niveles de consistencia temporal aceptables por ambos sexos (Sprecher y Metts, 1999). La escala cuenta con un total de 15 ítems divididos en cuatro subescalas etiquetadas del siguiente modo: 1) El amor encuentra un camino, 2) Uno y solo uno, 3) Idealización y 4) Amor a primera vista. Las respuestas a estos ítems se puntúan en una escala de tipo Likert en donde 1 es "en total desacuerdo" y 7 "totalmente de acuerdo". En la pregunta 10 del cuestionario (anexo 1) se incluyen todos los ítems. Como se explica en el siguiente subepígrafe (3.3), en nuestro trabajo se han reducido a 13 los ítems de esta escala por mayor carácter explicativo de los factores que la componen.

Además, y para medir si el alumnado de CAV justifica la violencia de género, incluimos en nuestro cuestionario los ítems contenidos en el factor I de la escala "Justificación del Sexismo y Violencia de Género" de Díaz-Aguado y Martínez Arias (2001). Este factor I denominado "Justificación de la violencia de género" está compuesto por 7 ítems con una alta consistencia interna (alpha de Cronbach=0,76). Las respuestas se enmarcan en una escala Likert del 1 ("nada de acuerdo) al 4 ("muy de acuerdo"). En la pregunta 11 del cuestionario (anexo 1) se incluyen todos los ítems de esta segunda escala. 
Además de las dos escalas utilizadas, como variables sociodemográficas del alumnado se tuvieron en cuenta: la edad y el sexo de los/as participantes; el curso de CAV al que pertenecían; si tenían o no pareja; el tipo de centro educativo donde habían estudiado; el estilo educativo de su familia; así como el nivel de estudios del padre y la madre.

Se adjunta el cuestionario con todas las variables estudiadas en el anexo1.

\subsection{Análisis de datos}

Nuestro análisis de datos se realizó mediante el programa estadístico IBM SPSS (v22.0), comenzando por un análisis exploratorio para identificar las características de la muestra. Además, y para comprobar si existen diferencias en torno a la interpretación de los mitos románticos en relación a las variables de sexo, tipo de centro educativo, modelo educativo familiar, nivel de estudios de los padres o curso, se implementa la prueba $t$ de Student para muestras independientes. Se entiende que un resultado es estadísticamente significativo cuando una probabilidad de cometer un error tipo I es de $p \leq 0.05$ (nivel de confianza del 95\%).

Por último, y aunque en el cuestionario aplicado hemos incluido la escala ROMBEL original (15 ítems), pretendemos validar este instrumento de medida para nuestra muestra, reduciendo el número de factores explicativos a través de un Análisis Factorial (se explica en el epígrafe 4 de "Resultados"). Antes, y para comprobar la pertinencia de este tipo de análisis, comprobamos la adecuación de la matriz de correlaciones con el cálculo del Índice de Kaiser-Meyer-Olkin (KMO) y la prueba de Bartlett $(\mathrm{p}=0,000)$. Nuestros análisis demuestran (tabla 3 ) una adecuación para realizar un análisis factorial, obteniendo un buen resultado de KMO $=0.822$.

Tabla 3. KMO y prueba de Bartlett

\begin{tabular}{|lll|}
\hline \multicolumn{2}{|l|}{ Medida de adecuación muestral de Kaiser-Meyer-Olkin. } &, 822 \\
Prueba de esfericidad de & Chi-cuadrado aproximado & 871,465 \\
Bartlett & gl & 105 \\
& Sig. &, 000 \\
\hline
\end{tabular}

Fuente: elaboración propia

Para evaluar si mantenemos o no todas las variables (ítems) de la escala ROMBEL original en nuestro análisis factorial, revisamos los valores de la "Matriz Anti-imagen" (y corroboramos con la "Tabla de Comunalidades"). En este sentido, comprobamos cómo claramente son dos variables las que menores valores presentan y serán las que eliminamos de nuestro modelo:

- AROMANTICO1: "No necesito conocer a alguien durante un tiempo antes de enamorarme de él o ella" (0.532).

- AROMANTICO2: "Si estuviera enamorado/a de alguien, me comprometería con él o ella aunque mis padres y amigos desaprobaran la relación” (0.641).

\section{Resultados}

\subsection{Mitos del amor romántico}

Aunque en el análisis original del test ROMBEL las autoras (Sprecher y Metts, 1989) obtuvieron una solución de cuatro factores, con nuestra muestra y tras la realización del análisis factorial (validado en el subepígrafe 3.3) se reduce el modelo a 3 factores que explican el $60,7 \%$ de la varianza total. La siguiente tabla (tabla 4) muestra la matriz de componentes rotada y sus correlaciones con cada factor, divididas para cada uno de los 3 factores de nuestro modelo:

Tabla 4. Matriz de componentes rotada y sus correlaciones

\section{Factor 1}

"Una vez que experimente el amor verdadero, nunca volveré a experimentarlo de nuevo con la misma intensidad, con otra persona" 
"Creo que estar verdaderamente enamorado es estarlo para siempre"

"Cuando encuentre mi "amor verdadero", probablemente lo sabré poco después de encontrarnos"

"Estoy seguro de que cada nuevo aspecto que conozca acerca de la persona elegida para un compromiso a largo plazo, me agradará"

"La relación que tenga con mi "amor verdadero" será casi perfecta"

"Habrá un solo amor verdadero para mí"

Factor 2

"Si amo a alguien, sé que puedo hacer que la relación funcione a pesar de las dificultades"

"Si amo a alguien, encontraré la forma de que estemos juntos con independencia de la oposición que exista, distancia física, o cualquier otra barrera

"Si la relación que mantengo es la que se supone tiene que ser, cualquier obstáculo (por ejemplo, falta de dinero, distancia física, conflictos en el trabajo) podrá superarse"

"Creo que si mi pareja y yo nos queremos, podemos superar cualquier problema que nos surja"

\section{Factor 3}

"Si encuentro a la persona adecuada, es muy probable que me enamore casi de inmediato"

"Espero que en mi relación de pareja, el amor romántico realmente dure; que no desaparezca con el tiempo"

“La persona a quien ame será la pareja romántica perfecta; por ejemplo, él/ella será tolerante, comprensivo/a y me amará"

Fuente: elaboración propia

Siguiendo la propia interpretación de las autoras de la escala ROMBEL combinada con la de literatura reciente (Bosch et al., 2007: 33-42; Fundación Mujeres, 2011: 7; Marmol-Martín, 2018: 57-58; RodriguezCastro, 2013: 165), nuestro modelo podría interpretarse clasificando cada factor en relación con uno o varios mitos del amor romántico tal y como se contiene en la siguiente tabla (tabla 5):

Tabla 5. Interpretación de factores en relación a los mitos del amor romántico

\begin{tabular}{|l|l|l|}
\cline { 2 - 3 } \multicolumn{1}{c|}{} & INTERPRETACIÓN & MITOS DE AMOR ROMÁNTICO \\
\hline FACTOR 1 & "El amor está predestinado" & $\begin{array}{l}\text { Mito de la media naranja } \\
\text { Creencia "un verdadero amor" para toda la vida } \\
\text { Mito de la perdurabilidad }\end{array}$ \\
\hline FACTOR 2 & "El amor todo lo puede" & Mito de la omnipotencia \\
\hline FACTOR 3 & "Para toda la vida" & $\begin{array}{l}\text { Mito de la equivalencia7 } \\
\text { Mito de la pasión eterna }\end{array}$ \\
\hline
\end{tabular}

Fuente: elaboración propia

A continuación (tabla 6), se analizan las diferentes interpretaciones de estos mitos según el perfil sociodemográfico del alumnado, subrayando únicamente aquellas variables en las que encontramos diferencias estadísticamente significativas (prueba $t$ de Student).

Creencia de que la pareja está predestinada por nacimiento.

El amor romántico y pasional puede perdurar a través del tiempo.

Creencia de que el amor lo puede todo y es suficiente para solucionar todos los problemas.

Creencia en que el amor -como sentimiento- y enamoramiento - como algo más superfluo y menos duradero-son equivalentes

Creencia de que el amor romántico y pasional de los primeros meses puede y debe perdurar eternamente. 
Tabla 6. Diferencias en los mitos del amor romántico según edad

\begin{tabular}{|c|c|c|c|c|c|}
\hline \multirow[b]{2}{*}{ Factor 1} & \multicolumn{2}{|c|}{ Hasta 20 años } & \multicolumn{2}{|c|}{ Más de 20 años } & \multirow{2}{*}{$t$} \\
\hline & M & DT & M & DT & \\
\hline $\begin{array}{l}\text { "Una vez que experimente el amor verdadero, nunca volveré a } \\
\text { experimentarlo de nuevo con la misma intensidad, con otra persona" }\end{array}$ & 2,31 & 1,61 & 2,62 & 1,806 & $-1,06$ \\
\hline "Creo que estar verdaderamente enamorado es estarlo para siempre" & 2,77 & 1,846 & 3,04 & 2,058 & $-0,803$ \\
\hline $\begin{array}{l}\text { "Cuando encuentre mi "amor verdadero", probablemente lo sabré poco } \\
\text { después de encontrarnos" }\end{array}$ & 2,6 & 1,426 & 2,69 & 1,732 & $-0,357$ \\
\hline $\begin{array}{l}\text { "Estoy seguro de que cada nuevo aspecto que conozca acerca de la } \\
\text { persona el egida para un compromiso a largo plazo, me agradará" }\end{array}$ & 2,99 & 1,829 & 2,52 & 1,686 & 1,537 \\
\hline "La relación que tenga con mi "amor verdadero" será casi perfecta" & 2,99 & 1,851 & 3 & 1,804 & $-0,033$ \\
\hline "Habrá un solo amor verdadero para mí" & 2,45 & 1,738 & 2,6 & 1,839 & $-0,468$ \\
\hline \multicolumn{6}{|l|}{ Factor 2} \\
\hline $\begin{array}{l}\text { "Si amo a alguien, sé que puedo hacer que la relación funcione a pesar de } \\
\text { las dificultades" }\end{array}$ & 4,4 & 1,807 & 3,94 & 1,924 & 1,447 \\
\hline $\begin{array}{l}\text { "Si amo a alguien, encontraré la forma de que estemos juntos con } \\
\text { independencia de la oposición que exista, distancia física, o cualquier } \\
\text { otra barrera }\end{array}$ & 4,76 & 1,755 & 4,37 & 1,826 & 1,3 \\
\hline $\begin{array}{l}\text { "Si la relación que mantengo es la que se supone tiene que ser, cualquier } \\
\text { obstáculo (por ejemplo, falta de dinero, distancia física, conflictos en el } \\
\text { trabajo) podrá superarse" }\end{array}$ & 4,84 & 1,869 & 4,6 & 1,983 & 0,728 \\
\hline $\begin{array}{l}\text { "Creo que si mi pareja y yo nos queremos, podemos superar cualquier } \\
\text { problema que nos surja" }\end{array}$ & 4,76 & 1,7 & 4,75 & 1,846 & 0,043 \\
\hline \multicolumn{6}{|l|}{ Factor 3} \\
\hline $\begin{array}{l}\text { "Si encuentro a la persona adecuada, es muy probable que me enamore } \\
\text { casi de inmediato" }\end{array}$ & 3,21 & 1,726 & 2,37 & 1,664 & $2,049 *$ \\
\hline $\begin{array}{l}\text { "Espero que en mi relación de pareja, el amor romántico realmente dure; } \\
\text { que no desaparezca con el tiempo" }\end{array}$ & 5,01 & 1,655 & 4,05 & 2,066 & $2,147^{*}$ \\
\hline $\begin{array}{l}\text { "La persona a quien ame será la pareja romántica perfecta; por ejemplo, } \\
\text { él/ella será tolerante, comprensivo/a y me amará" }\end{array}$ & 4,29 & 2,005 & 3,79 & 1,993 & 1,454 \\
\hline
\end{tabular}

$* \mathrm{p}<0.05$

Entendiendo que la variable "curso" podría no ser determinante en la edad del alumnado, preferimos hacer uso de la variable "edad" para evaluar las posibles diferencias en la interpretación de los mitos del amor romántico entre el alumnado. Así, se observa en la tabla 6 cómo es el alumnado más joven quienes siguen manteniendo mitos en torno a la omnipotencia del amor. En concreto, encontramos diferencias estadísticamente significativas en dos de los tres ítems de nuestro factor 3 ("Si encuentro a la persona adecuada, es muy probable que me enamore casi de inmediato" y "Espero que en mi relación de pareja, el amor romántico realmente dure; que no desaparezca con el tiempo").

Este hallazgo nos permite confirmar nuestra hipótesis 3 (H3: Es el alumnado más joven quien en mayor medida perpetúa algunos mitos del amor romántico). 
Tabla 7. Diferencias en los mitos del amor romántico según "tener o no pareja"

\begin{tabular}{|c|c|c|c|c|c|}
\hline \multirow[b]{2}{*}{ Factor 1} & \multicolumn{2}{|c|}{ Con pareja } & \multicolumn{2}{|c|}{ Sin pareja } & \multirow{2}{*}{$t$} \\
\hline & $\mathrm{M}$ & DT & $\mathrm{M}$ & DT & \\
\hline $\begin{array}{l}\text { "Una vez que experimente el amor verdadero, nunca volveré a } \\
\text { experimentarlo de nuevo con la misma intensidad, con otra persona" }\end{array}$ & 2,47 & 1,676 & 2,38 & 1,692 & 0,314 \\
\hline "Creo que estar verdaderamente enamorado es estarlo para siempre" & 3,00 & 1,944 & 2,79 & 1,911 & 0,652 \\
\hline $\begin{array}{l}\text { "Cuando encuentre mi "amor verdadero", probablemente lo sabré poco } \\
\text { después de encontrarnos" }\end{array}$ & 2,47 & 1,438 & 2,72 & 1,589 & $-0,962$ \\
\hline $\begin{array}{l}\text { "Estoy seguro de que cada nuevo aspecto que conozca acerca de la } \\
\text { persona el egida para un compromiso a largo plazo, me agradará" }\end{array}$ & 2,71 & 1,641 & 2,89 & 1,875 & $-0,606$ \\
\hline "La relación que tenga con mi "amor verdadero" será casi perfecta" & 3,27 & 1,91 & 2,83 & 1,77 & 0,34 \\
\hline "Habrá un solo amor verdadero para mí" & 2,69 & 1,855 & 2,39 & 1,718 & 0,99 \\
\hline \multicolumn{6}{|l|}{ Factor 2} \\
\hline $\begin{array}{l}\text { "Si a mo a alguien, sé que puedo hacer que la relación funcione a pesar de } \\
\text { las dificultades" }\end{array}$ & 4,44 & 1,922 & 4,13 & 1,815 & 0,98 \\
\hline $\begin{array}{l}\text { "Si amo a alguien, encontraré la forma de que estemos juntos con } \\
\text { independencia de la oposición que exista, distancia física, o cualquier } \\
\text { otra barrera" }\end{array}$ & 4,96 & 1,784 & 4,12 & 1,763 & $1,96^{*}$ \\
\hline $\begin{array}{l}\text { "Si la relación que mantengo es la que se supone tiene que ser, cualquier } \\
\text { obstáculo (por ejemplo, falta de dinero, distancia física, conflictos en el } \\
\text { trabajo) podrá superarse" }\end{array}$ & 5,09 & 1,869 & 4,55 & 1,91 & 1,672 \\
\hline $\begin{array}{l}\text { "Creo que si mi pareja y yo nos queremos, podemos superar cualquier } \\
\text { problema que nos surja" }\end{array}$ & 4,91 & 1,878 & 4,67 & 1,668 & 0,805 \\
\hline \multicolumn{6}{|l|}{ Factor 3} \\
\hline $\begin{array}{l}\text { "Si encuentro a la persona adecuada, es muy probable que me enamore } \\
\text { casi de inmediato" }\end{array}$ & 3,09 & 1,735 & 3,03 & 1,707 & 0,202 \\
\hline $\begin{array}{l}\text { "Espero que en mi relación de pareja, el amor romántico realmente dure; } \\
\text { que no desaparezca con el tiempo" }\end{array}$ & 5,27 & 1,737 & 4,66 & 1,823 & $2,016^{*}$ \\
\hline $\begin{array}{l}\text { "La persona a quien ame será la pareja romántica perfecta; por ejemplo, } \\
\text { él/ella será tolerante, comprensivo/a y me amará" }\end{array}$ & 4,51 & 1,971 & 3,68 & 2,005 & $1,91^{*}$ \\
\hline
\end{tabular}

Observamos cómo también se hallan diferencias estadísticamente significativas en función de estar o no emparejado (tabla 7), en dos de los 3 ítems del factor 3 "Para toda la vida" ("Espero que en mi relación de pareja, el amor romántico realmente dure; que no desaparezca con el tiempo" y "La persona a quien ame será la pareja romántica perfecta; por ejemplo, él/ella será tolerante, comprensivo/a y me amará"). Es aquél alumnado con pareja quien perpetúa estos mitos, estando también presente, aunque en menor medida, el mito de la omnipotencia del amor ("Si amo a alguien, encontraré la forma de que estemos juntos con independencia de la oposición que exista, distancia física o cualquier otra barrera"). 
Tabla 8- Diferencias en los mitos del amor romántico según "tipo de centro educativo"

\begin{tabular}{|c|c|c|c|c|c|}
\hline \multirow[b]{2}{*}{ Factor 1} & \multicolumn{2}{|c|}{ Colegio público } & \multicolumn{2}{|c|}{ Colegio privado } & \multirow{2}{*}{$t$} \\
\hline & $\mathrm{M}$ & DT & $\mathrm{M}$ & DT & \\
\hline $\begin{array}{l}\text { "Una vez que experimente el amor verdadero, nunca volveré a } \\
\text { experimentarlo de nuevo con la misma intensidad, con otra persona" }\end{array}$ & 2,44 & 1,749 & 2,37 & 1,585 & 0,253 \\
\hline "Creo que estar verdaderamente enamorado es estarlo para siempre" & 2,76 & 1,945 & 3,03 & 1,884 & $-0,865$ \\
\hline $\begin{array}{l}\text { "Cuando encuentre mi "amor verdadero", probablemente lo sabré poco } \\
\text { después de encontrarnos" }\end{array}$ & 2,57 & 1,558 & 2,73 & 1,507 & $-0,629$ \\
\hline $\begin{array}{l}\text { "Estoy seguro de que cada nuevo aspecto que conozca acerca de la } \\
\text { persona el egida para un compromiso a largo plazo, me agradará" }\end{array}$ & 2,53 & 1,671 & 3,27 & 1,883 & $.-2,506^{*}$ \\
\hline "La relación que tenga con mi "amor verdadero" será casi perfecta" & 2,87 & 1,874 & 3,19 & 1,757 & $-1,044$ \\
\hline "Habrá un solo amor verdadero para mí" & 2,46 & 1,861 & 2,58 & 1,632 & $-0,406$ \\
\hline \multicolumn{6}{|l|}{$\begin{array}{r}\text { Factor } 2 \\
\end{array}$} \\
\hline $\begin{array}{l}\text { "Si amo a alguien, sé que puedo hacer que la relación funcione a pesar de } \\
\text { las dificultades" }\end{array}$ & 3,97 & 1,833 & 4,66 & 1,825 & $.-2,265^{*}$ \\
\hline $\begin{array}{l}\text { "Si amo a alguien, encontraré la forma de que estemos juntos con } \\
\text { independencia de la oposición que exista, distancia física, o cualquier } \\
\text { otra barrera" }\end{array}$ & 4,49 & 1,856 & 4,83 & 1,662 & $-1,144$ \\
\hline $\begin{array}{l}\text { "Si la relación que mantengo es la que se supone tiene que ser, cualquier } \\
\text { obstáculo (por ejemplo, falta de dinero, distancia física, conflictos en el } \\
\text { trabajo) podrá superarse" }\end{array}$ & 4,7 & 1,997 & 4,83 & 1,773 & $-0,408$ \\
\hline $\begin{array}{l}\text { "Creo que si mi pareja y yo nos queremos, podemos superar cualquier } \\
\text { problema que nos surja" }\end{array}$ & 4,57 & 1,824 & 5,25 & 1,591 & $.-1,885^{*}$ \\
\hline \multicolumn{6}{|l|}{ Factor 3} \\
\hline $\begin{array}{l}\text { "Si encuentro a la persona adecuada, es muy probable que me enamore } \\
\text { casi de inmediato" }\end{array}$ & 3,02 & 1,702 & 3,1 & 1,739 & $-0,276$ \\
\hline $\begin{array}{l}\text { "Espero que en mi relación de pareja, el amor romántico realmente dure; } \\
\text { que no desaparezca con el tiempo" }\end{array}$ & 4,83 & 1,844 & 4,97 & 1,771 & $-0,437$ \\
\hline $\begin{array}{l}\text { "La persona a quien ame será la pareja romántica perfecta; por ejemplo, } \\
\text { él/ella será tolerante, comprensivo/a y me amará" }\end{array}$ & 4,1 & 2,094 & 4,14 & 1,889 & $-0,105$ \\
\hline
\end{tabular}

$* \mathrm{p}<0.05$

La anterior tabla (tabla 8) pone de manifiesto cómo se encuentran igualmente algunas diferencias estadísticamente significativas según el "tipo de centro educativo" en el que estudió el alumnado de CAV. Así, vemos cómo es aquél alumnado cuyo proceso de socialización primaria se enmarcó en un centro educativo privado, quien mantiene algunos mitos de la omnipotencia del amor. Encontramos diferencias significativas en dos de los 4 ítems de nuestro factor 2 "El amor todo lo puede", en concreto: "Si amo a alguien, sé que puedo hacer que la relación funcione a pesar de las dificultades" y "Creo que si mi pareja y yo nos queremos, podemos superar cualquier problema que nos surja". Este último se ve reforzado cuando el centro educativo, además de privado es religioso $\left(t=-1.950^{*}\right)$. Del mismo modo, se comprueba cómo el alumnado del sistema educativo privado parece perpetuar todavía el "mito de la perdurabilidad" enmarcado en nuestro modelo dentro de nuestro factor 1 ("Estoy seguro de que cada nuevo aspecto que conozca acerca de la persona elegida para un compromiso a largo plazo, me agradará").

Este hallazgo nos permite confirmar nuestra hipótesis 4 (H4: El tipo de centro educativo en el que ha estudiado el alumnado universitario de CAV provoca diferentes interpretaciones del amor romántico).

Por lo que respecta a la influencia de la familia en la socialización primaria del alumnado en torno a la interpretación de los mitos del amor romántico, se hallan diferencias estadísticamente significativas tanto en relación al nivel de estudios de los padres como con respecto al "estilo educativo" del núcleo familiar.

Así, cuanto menor sea el nivel de estudios del padre, mayor fuerza adquiere la sensación entre el alumnado de que "El amor está predestinado" (factor 1) donde se perpetúan mitos como el de la "media naranja" o la "perdurabilidad" del amor. Estos hallazgos se ponen de manifiesto en la siguiente tabla (tabla 9): 
Tabla 9. Diferencias en los mitos del amor romántico según "nivel de estudios del padre"

\begin{tabular}{|c|c|c|c|c|c|}
\hline \multirow[b]{2}{*}{ Factor 1} & \multicolumn{2}{|c|}{$\begin{array}{l}\text { Hasta estudios } \\
\text { secundarios }\end{array}$} & \multicolumn{2}{|c|}{ Estudios superiores } & \multirow[t]{2}{*}{$t$} \\
\hline & M & DT & M & DT & \\
\hline $\begin{array}{l}\text { "Una vez que experimente el amor verdadero, nunca volveré a } \\
\text { experimentarlo de nuevo con la misma intensidad, con otra persona" }\end{array}$ & 2,73 & 1,954 & 2,09 & 1,284 & $2,354^{*}$ \\
\hline "Creo que estar verdaderamente enamorado es estarlo para siempre" & 3,11 & 2,017 & 2,62 & 1,796 & 1,549 \\
\hline $\begin{array}{l}\text { "Cuando encuentre mi "amor verdadero", probablemente lo sabré poco } \\
\text { después de encontrarnos" }\end{array}$ & 2,88 & 1,7 & 2,38 & 1,311 & $2,015^{*}$ \\
\hline $\begin{array}{l}\text { "Estoy seguro de que cada nuevo aspecto que conozca acerca de la } \\
\text { persona elegida para un compromiso a largo plazo, me agradará" }\end{array}$ & 3,00 & 1,808 & 2,65 & 1,763 & 1,201 \\
\hline "La relación que tenga con mi "amor verdadero" será casi perfecta" & 3,31 & 1,881 & 2,68 & 1,729 & $2,131^{*}$ \\
\hline "Habrá un solo amor verdadero para mí" & 2,71 & 1,937 & 2,3 & 1,568 & 1,417 \\
\hline \multicolumn{6}{|l|}{ Factor 2} \\
\hline $\begin{array}{l}\text { "Si amo a alguien, sé que puedo hacer que la relación funcione a pesar de } \\
\text { las dificultades" }\end{array}$ & 4,11 & 1,871 & 4,38 & 1,841 & $-0,893$ \\
\hline $\begin{array}{l}\text { "Si a mo a alguien, encontraré la forma de que estemos juntos con } \\
\text { independencia de la oposición que exista, distancia física, o cualquier } \\
\text { otra barrera" }\end{array}$ & 4,6 & 1,831 & 4,65 & 1,747 & $-0,166$ \\
\hline $\begin{array}{l}\text { "Si la relación que mantengo es la que se supone tiene que ser, cualquier } \\
\text { obstáculo (por ejemplo, falta de dinero, distancia física, conflictos en el } \\
\text { trabajo) podrá superarse" }\end{array}$ & 4,81 & 1,908 & 4,69 & 1,916 & 0,396 \\
\hline $\begin{array}{l}\text { "Creo que si mi pareja y yo nos queremos, podemos superar cualquier } \\
\text { problema que nos surja" }\end{array}$ & 4,84 & 1,725 & 4,68 & 1,776 & 0,573 \\
\hline \multicolumn{6}{|l|}{ Factor 3} \\
\hline $\begin{array}{l}\text { "Si encuentro a la persona adecuada, es muy probable que me enamore } \\
\text { casi de inmediato" }\end{array}$ & 3,28 & 1,871 & 2,82 & 1,511 & 1,634 \\
\hline $\begin{array}{l}\text { "Espero que en mi relación de pareja, el amor romántico realmente dure; } \\
\text { que no desaparezca con el tiempo" }\end{array}$ & 5,27 & 1,671 & 4,5 & 1,874 & $2,636^{*}$ \\
\hline $\begin{array}{l}\text { "La persona a quien ame será la pareja romántica perfecta; por ejemplo, } \\
\text { él/ella será tolerante, comprensivo/a y me amará" }\end{array}$ & 4,32 & 2,021 & 3,91 & 1,987 & 1,262 \\
\hline
\end{tabular}

$$
* \mathrm{p}<0.05
$$

El nivel de estudios de la madre no ha resultado estadísticamente significativo en la interpretación de estos mitos, a excepción de dos ítems relacionados con el "amor verdadero" ("Habrá un solo amor verdadero para mí", $t=2,036^{*}$ ) y la perdurabilidad del amor ("Espero que en mi relación de pareja, el amor romántico realmente dure; que no desaparezca con el tiempo", $\left.t=2,359^{*}\right)$. A menor nivel de estudios de la madre del alumno/a, mayor perpetuación de estas ideas.

Y por último, también en el "estilo educativo familiar" del alumnado encontramos un par de diferencias en torno a la asunción de estos mitos en torno al amor. Recodificando nuestra variable original en estilo autoritario y no autoritario 9 , nuestros datos ponen de manifiesto como es el alumnado con familias "autoritarias" quienes siguen perpetuando algunos mitos como el de la perdurabilidad del amor ("Creo que estar verdaderamente enamorado es estarlo para siempre", $t=2.216^{*}$; "Espero que en mi relación de pareja, el amor romántico realmente dure; que no desaparezca con el tiempo", $\left.t=1.945^{*}\right)$.

\subsection{Justificación de la violencia de género}

Con el fin de medir si el alumnado de CAV justifica o no la violencia de género, hacemos uso del factor I denominado "Justificación de la violencia de género" (Díaz-Aguado y Martínez Arias, 2001) compuesto por 7 ítems.

Como se puede observar en la siguiente tabla (tabla 9), encontramos un amplio rechazo a la justificación de la violencia de género entre nuestro alumnado de CAV. En una escala acuerdo de 1 (nada de acuerdo) a 4 (muy de acuerdo), la media con todos los ítems ronda el valor más pequeño y apenas existe dispersión con respecto al valor medio. 
Tabla 9. Descriptivos variables "justificación violencia de género"

\begin{tabular}{|l|c|c|}
\cline { 2 - 3 } \multicolumn{1}{c|}{} & Media & Desviación típica \\
\hline $\begin{array}{l}\text { Grado acuerdo "Por el bien de sus hijos, aunque la mujer ten- } \\
\text { ga que soportar la violencia de su marido o compañero con- } \\
\text { viene que no le denuncie" }\end{array}$ & 1,05 &, 324 \\
\hline $\begin{array}{l}\text { Grado acuerdo "Si una mujer es maltratada por su compañe- } \\
\text { ro y no le abandona será porque no le disgusta del todo esa } \\
\text { situación" }\end{array}$ & 1,06 &, 239 \\
\hline $\begin{array}{l}\text { Grado acuerdo "Cuando una mujer es agredida por su marido, } \\
\text { algo habrá hecho ella para provocarlo" }\end{array}$ & 1,08 &, 319 \\
\hline $\begin{array}{l}\text { Grado acuerdo "Un buen padre debe hacer saber al resto de su } \\
\text { familia quién es el que manda" }\end{array}$ & 1,07 &, 342 \\
\hline $\begin{array}{l}\text { Grado acuerdo "Para tener una buena relación es deseable que } \\
\text { la mujer evite llevar la contraria al hombre" }\end{array}$ & 1,04 &, 282 \\
\hline $\begin{array}{l}\text { Grado acuerdo "La violencia que se produce dentro de casa es } \\
\text { un asunto de la familia y no debe salir de ahí" }\end{array}$ & 1,08 &, 297 \\
\hline $\begin{array}{l}\text { Grado acuerdo "Está justificado que un hombre agreda a su } \\
\text { mujer o a su novia cuando ella decide dejarle" }\end{array}$ & 1,02 &, 183 \\
\hline
\end{tabular}

Fuente: elaboración propia

Aunque estos resultados descriptivos anticipan un amplio rechazo de toda nuestra muestra a la violencia de género, se han analizado igualmente las posibles diferencias de género (hombre-mujer) o en otras variables sociodemográficas en torno a este tema (prueba $t$ de Student). Sin embargo, los análisis reafirman cómo el rechazo es conjunto, independientemente del perfil del alumno/a (sexo, edad, curso, tipo de centro educativo, estilo educativo familiar o nivel de estudios de los padres).

Este hallazgo nos permite confirmar nuestra hipótesis 1 (H1: El alumnado universitario de CAV no justifica la violencia de género, independientemente de su sexo).

\section{Conclusiones y discusión}

Los resultados de esta investigación ponen de manifiesto la vigencia de algunos mitos del amor romántico en una muestra de futuras y futuros encargados de diseñar las relaciones amorosas en el mundo audiovisual.

El sexo del alumnado ${ }^{10}$ no determina diferente asunción de estos mitos, sino que son otras variables sociodemográficas las que están influyendo. Así, la edad del alumnado sí muestra diferentes interpretaciones del amor romántico. En concreto, es el alumnado más joven (menores de 20 años) quien sigue manteniendo mitos en torno a la "omnipotencia" del amor. También el hecho de tener pareja es una variable determinante para perpetuar algunos mitos, en concreto, el alumnado con pareja es quien más defiende la "perdurabilidad" del amor y en parte, también su omnipotencia.

Si bien nuestro alumnado va a convertirse en el futuro próximo en agentes de socialización de género (en tanto que parte de los medios), ellos/as en cuanto a individuos han estado influenciados por otros agentes clave como son el centro educativo y la familia. De hecho, Mosterio y Porto (2017: 154) confirman cómo la escuela a través del currículum oculto, refuerza los estereotipos de género que se inician en el seno familiar.

Así, nuestros datos muestran cómo los alumnos/as que cursaron sus estudios en un centro educativo privado mantienen algunos mitos de la omnipotencia y la perdurabilidad del amor. Del mismo modo, tanto el estilo educativo familiar como el nivel de estudios de los padres, ponen de manifiesto cómo también parece estar influyendo en la socialización primaria del alumnado de CAV en torno a la interpretación de los mitos del amor romántico. Cuanto menor sea el nivel de estudios del padre (y de la madre, en menor medida), mayor fuerza adquiere la sensación entre el alumnado de que "El amor está predestinado". Y es aquél alumnado con familias autoritarias quien perpetúa en cierta medida el mito en torno a la perdurabilidad del amor.

Sin embargo, un hecho a subrayar, es que no encontramos diferencias de género en estas interpretaciones contradiciendo a las conclusiones de otros estudios de carácter tanto cuantitativo como cualitativo realizados para el caso español (Bosch et al., 2007; Caro y Monreal, 2017; Rodríguez y Alonso, 2015; Rodríguez-Castro et al., 2013).

También es importante resaltar cómo se expone un rotundo rechazo por parte del alumnado de CAV en torno a la violencia de género. Este hallazgo es sumamente importante, sobre todo confirmando la inexistencia

Hallazgo que nos hace tener que refutar nuestra hipótesis de estudio 2 (H2: Encontramos diferencias de género en la interpretación de los mitos del amor romántico). 
de diferencias entre alumnos y alumnas, ya que otros estudios previos (Díaz-Aguado y Carvajal, 2012: 255) sí confirmaban cómo los alumnos universitarios (chicos) justificaban este tipo de violencia aunque con diferente instrumento de medida.

En conclusión, en nuestra muestra de estudio siguen presentes en cierta medida los mitos de perdurabilidad y omnipotencia del amor entre los/las más jóvenes, con pareja, de centro educativo privado y con familias autoritarias o con padres con bajo nivel de estudios. Sin embargo, parece que el alumnado de CAV deconstruye muchos de los mitos más "peligrosos" en torno al amor romántico como son el de la media naranja o los celos, y rechazan de forma unánime la violencia de género. Estos hallazgos nos permiten vislumbrar una posible ruptura de algunos estereotipos de género en torno al diseño de las relaciones amorosas en películas, series u otros productos audiovisuales de los que sean responsables.

Sin embargo, no cabe duda por los resultados que hay que seguir trabajando en la socialización primaria de género, en donde agentes como la familia y la escuela son clave. Y del mismo modo, también es importante la formación en género en el marco de la Educación Superior puesto que, como afirman Caro y Monreal (2017: 54), es una herramienta eficaz para deconstruir creencias aprendidas sobre el 'mal amor' y completar el desarrollo de 'conciencia de género'. De hecho, Menéndez (2013: 702; 2014: 23) subraya cómo incluso tras la culminación del Plan Bolonia, apenas se han incorporado contenidos curriculares de género en la formación universitaria ni siquiera en los estudios de comunicación (Publicidad y Relaciones Públicas, Comunicación Audiovisual y Periodismo), donde la perspectiva de género es casi inexistente a pesar del rol hegemónico de los medios.

Por último, cabe apuntar que todos estos hallazgos deben interpretarse con las limitaciones del tamaño muestral, que se tratará de subsanar replicando el estudio en posteriores oleadas para ampliar la muestra de estudio.

\section{Referencias bibliogfáficas}

Aron, A., Fisher, H. y Mashek, D.J. (2005). "Reward, motivation, and emotion systems associated with early-stage intense romantic love". En: Journal of Neurophisiology, 94(1), pp. 327-337. doi: 10.1152/jn.00838.2004

Barrón, A., Martínez-Iñigo, D., Paúl, P. y Yela, C. (1999). "Romantic beliefs and myths in Spain". En: The Spanish Journal of Psychology, 2, pp. 64-73. doi: 10.1017/S1138741600005461

Bonilla-Algovia, E. y Rivas-Rivero, E. (2018). "Mitos del amor romántico en una muestra de futuros y futuras docentes". En: Revista Estudios e Investigación en Psicología y Educación, 5(2), pp. 113-117. doi: https://doi.org/10.17979/ reipe.2018.5.2.3624

Bernárdez, A., García, I. y Gonzánlez, S. (2008). Violencia de género en el cine español. Madrid: Universidad Complutense.

Bosch, E., Ferrer, V., García, E., Ramis, M.c., Mas, M.c., Navarro, C. y Torrens, G. (2007). Del mito del amor romántico a la violencia contra las mujeres en la pareja. Madrid: Instituto de La Mujer. Ministerio de Igualdad.

Bradbury, T. N. y Fincham, F. D. (1990). "Attributions in marriage: Review and critique”. En: Psychological Bulletin, 107 (3). doi: 10.1037//0033-2909.107.1.3

Caro, C. y Monreal, M.C. (2017). “Creencias del amor romántico y violencia de género”. En: INFAD Revista de Psicología, 1- Monográfico 1, pp. 47-56. doi: https://doi.org/10.17060/ijodaep.2017.n1.v2.917

Carreño, M. y Serrano, G. (1995). “Análisis de instrumentos para la medida del amor”. En: Revista de Psicologia Social, 10, pp. 131-148. doi: 10.1174/021347495763810938

Chan, B. y Xueli, W. (2011). "Of prince charming and male chauvinist pigs: Singaporean female viewers and dreamworld of Korean televisión dramas". International Journal of Cultural Studies, 14(3), pp. 291-305.

Díaz-Aguado, M. J. y Martínez-Arias, R. (2001). "La construcción de la igualdad y la prevención de la violencia contra la mujer desde la educación secundaria". En: Instituto de la Mujer. Serie estudios $\mathrm{n}^{\circ}$ 73. Madrid.

Díaz-Aguado, M. J. y Carvajal, M. I. (2012). La juventud universitaria ante la igualdad y la violencia de género. Madrid: Ministerio de Sanidad, Igualdad y Servicios Sociales.

Duque, E. (2010-2011). Miratge de l'ascens i amor ideal. Noves propostes preventives de la violencia masclista. Barcelona: Intituto Catalán de la Mujer. Gobierno de Cataluña.

Esteban, M. L. y Távora, A. (2008). "El amor romántico y la subordinación social de las mujeres: revisiones y propuestas". En: Anuario de Psicología, 39, pp. 59-73.

Falcón, L. y Díaz-Aguado, M. J. (2014). "Relatos audiovisuales de ficción sobre la identidad adolescente en contextos escolares". En: Comunicar, 42, pp. 147-155.

Fernández-Enguita, M. (2014). "¿Cómo salvar de sí misma a la universidad? Retos y límites de la profesión académica”. En: RIS, 72(1), pp. 203-248. doi: 10.3989/ris.2014.i1.557

Fernández, A. y Navas, M. (2016). "Creencias sexistas y percepción de abuso en mujeres adolescentes y adultas". En: Raudem, Revista de Estudios de las Mujeres, Vol. 4. doi: http://dx.doi.org/10.25115/raudem.v4i0.1736

Ferrer, V., Bosch, E. y Navarro, C. (2010). "Los mitos románticos en España”. En: Boletín de Psicología, 99, pp. 7-31.

ferrer, V., Bosch, E. y Navarro, C. y Ferreiro, V. (2010). "El mito romántico de los celos y su aceptación en la sociedad española actual”. En: Apuntes De Psicología, 28, pp. 391-402. 
Ferris, A. L., Smith, S. W., Greenberg, B. S. y Smith, S. L. (2007). "The content of reality dating shows and viewer perceptions of dating". En: Journal of Communication, 57, pp. 490-510. doi: 0.1111/j.1460-2466.2007.00354.x

Fundación Mujeres (2011). "Mitos del amor romántico y prevención de la violencia de género". En: Monográfico 93. Coeducación y mitos del amor romántico. Disponible en: http:/www.fundacionmujeres.es/files/attachments/ Documento/46001/image/_BOLETIN\%20FM\%2093.pdf

García-Cueto, E., Rodríguez-Díaz, F.j., Bringas-Molleda, C., López-Cepedo, J., Paíno-Quesada, S. y Rodríguez-Franco, L. (2015). "Development of the Gender Role Attitudes Scale (GRAS) amongst young Spanish people". En: International Journal of Clinical and Health Psychology, 15, pp. 61-68. doi: 10.1016/j.ijchp.2014.10.004

Giddens, A. (2001). Sociología. Madrid: Alianza Editorial.

Grande, I. y Abascal, E. (2005). Análisis de encuestas. Madrid: ESIC.

Jones, J., y Cunnigham, J. (1996). "Attachment styles and other predictors of relationship satisfaction in dating couples". En: Personal Relationships, 3, pp. 387-399. doi: 10.1111/j.1475-6811.1996.tb00123.x

Hefner, V. y Wilson, B. J. (2013). "From Love at First Sight to Soul Mate: The Influence of Romantic Ideals in Popular Films on Young People's Beliefs about Relationships”. En: Communication Monographs, 80(2), pp. 150-175. doi: 10.1080/03637751.2013.776697

Hyman, H. (1942). “The Psychology of Status”. En: Archives of Psychology, 269, pp. 5-86.

Lee, L. J. (2008). “Guilty pleasures: Reading romance novels as reworked fairy tales”. En: Marvels and Tales, 22, pp. 52-66.

Marmol-Martín, I., Mena-Vega, S. y Rebollo-Bueno, S. (2018). "El amor romántico en los productos audiovisuales de ficción". En: Revista Admira, 6, pp. 52-81.

McMillan, J. H. y Schumacher, S. (2005). Investigación educativa. Madrid: Pearson Addison Wesley.

Menéndez, M. I. (2013). "Metodologías de innovación docente: la perspectiva de género en Comunicación Audiovisual”. En: Historia y Comunicación Social, Vol. 18 N Especial Octubre, pp. 699-710.

- (2014). El Espacio Europeo de Educación Superior en España: incorporación de contenidos y metodologías de género en comunicación”. En: Revista Internacional de Comunicación y Desarrollo, 1 (0), pp. 23-34.

Moreno, M., González, A. y Ros, M. (2007). “Enamoramiento y violencia contra las mujeres”. En: V.A. Ferrer y E. Bosch (Comps.), Los feminismos como herramientas de cambio social (II): De la violencia contra las mujeres a la construcción del pensamiento feminista, pp. 21-34. Palma de Mallorca: UIB.

Mosterio-García, M.J. Yy Porto-Castro, A.M. (2017). "Análisis de los estereotipos de género en alumnado de formación profesional: diferencias según sexo, edad y grado”. En: Revista de Investigación Educativa, 35(1), pp. 151-165. doi: http://dx.doi.org/10.6018/rie.35.1.257191

Nabi, R., Finnerty, K., Domschke, T. y Hull, S. (2006). "Does misery love company? Exploring the therapeutic effects of TV viewing on regretted experiences". En: Journal of Communication, 56, pp. 689-706. doi: 10.1111/j.14602466.2006.00315.x

Neves, A. S. (2007). “As mulheres e os discursos genderizados sobre o amor: a caminho do «amor confluente» ou o retorno ao mito do «amor romântico»?”. En: Revista Estudos Feministas, 15, (3), pp. 609-627.

Rodríguez-Castro, Y., Lameiras-Fernández, M., Carrera-Fernández, M.V. y Vallejo-Medina, P. (2013). "La fiabilidad y validez de la escala de mitos hacia el amor: las creencias de los y las adolescentes". En: Revista de Psicología Social, 28 (2), pp. 157-168. doi: 10.1174/021347413806196708

Rodríguez-Santero, J., García-Carpintero, M. A. y Porcel-Gálvez, A. M. (2017). "Los estilos de amor en estudiantes universitarios. Diferencias en función del sexo-género”. En: Revista Internacional de Sociología, 75 (3), e073. doi: http://dx.doi.org/10.3989/ris.2017.75.3.15.171

Sharp, E., y Ganong, L. (2000). "Raising awareness about marital expectations: Are unrealistic beliefs changed by integrative teaching?”. En: Family Relations, 49, pp. 71-76. doi: 10.1111/j.1741-3729.2000.00071.x

Sprecher, S. y Metts, S. (1989). "Development of the "romantic beliefs scale" and examination of the effects of gender and gender-role orientation". En: Journal of Social and Personal Relationships, 6 (4), pp. 387-411. doi: 10.1177/0265407589064001

- (1999). "Romantics beliefs: Their influence on relationships and patterns of change over time". En: Journal of Social and Personal Relationships, 16, pp. 834-851. doi: 10.1177/0265407599166009

Stone, L. (1989). "Passionate attachments in the West in historical perspective". En: W. GAYLIN y E. PERSON (Eds.), Pasionate attachments: thinking about love, pp. 15-27. Nueva York: Touchstone.

Tajahuerce, I., y Ramírez, E. (Eds.). (2018). La intervención en violencia de género desde diversos ámbitos. Madrid: Dykinson.

Winn, L. (2007). "Carpe diem: Relational scripts and "seizing the day" in the Hollywood romantic comedy". En: M. L. Galician y D. L. Merskin (Eds.), Critical thinking about sex, love, and romance in the mass media, pp. $247-264$. Mahwah: Lawrence Erlbaum Associates.

Yela, C. (2000). El amor desde la psicología social: Ni tan libres, ni tan racionales. Madrid: Pirámide.

- (2003). "La otra cara del amor: Mitos, paradojas y problemas”. En: Encuentros En Psicología Social, 1, pp. $263-267$.

Yuste, M., Serrano, M. A., Girbés, D. y Arandia, M. (2014). "Romantic Love and Gender Violence Clarifying Misunderstandings Through Communicative Organization of the Research". En: Qualitative Inquiry, 20(7), pp. 850855. doi: $10.1177 / 1077800414537206$ 


\section{Anexos}

\subsection{Anexo 1: Cuestionario}

1. Curso:
a. Primero
b. Segundo
c. Tercero
d. Cuarto

2. Sexo:
a. Mujer
b. Hombre
c. Otro

3. Edad: (abierta)

4. ¿Tiene pareja actualmente?
a. Sí
b. No

5. ¿En qué tipo de colegio/instituto estudió la ESO?
a. Público
b. Privado o concertado laico
c. Privado o concertado religioso

6. ¿Cuál es el nivel de estudios de su padre?
a. No acabó estudios primarios
b. Educación Primaria
c. Educación Secundaria Obligatoria, Bachillerato o FP
d. Diplomatura o carrera media
e. Licenciatura universitaria
f. Doctorado o Máster

7. ¿Cuál es el nivel de estudios de su madre?
a. No acabó estudios primarios
b. Educación Primaria
c. Educación Secundaria Obligatoria, Bachillerato o FP
d. Diplomatura o carrera media
e. Licenciatura universitaria
f. Doctorado o Máster

8. ¿Cómo definiría el estilo educativo de su familia?
a. Democrático
b. Autoritario
c. Permisivo

9. ¿Cuánto de influyente considera el papel del periodismo, la publicidad o los productos audiovisuales en las relaciones amorosas reales?
a. Mucho
b. Bastante
c. Poco
d. Nada

10.Indica el grado de acuerdo o desacuerdo con cada una de las siguientes creencias (teniendo en cuenta que:

Totalmente de acuerdo $=7, \mathrm{Ni}$ acuerdo ni en desacuerdo $=4$, Totalmente en desacuerdo $=1$ ).

10.1. No necesito conocer a alguien durante un tiempo antes de enamorarme de él o ella

10.2. Si estuviera enamorado/a de alguien, me comprometería con él o ella aunque mis padres y amigos/as desaprobaran la relación.

10.3. Una vez que experimente el amor verdadero, nunca volveré a experimentarlo de nuevo con la misma intensidad, con otra persona.

10.4. Creo que estar verdaderamente enamorado es estarlo para

10.5. siempre. Si amo a alguien, sé que puedo hacer que la relación funcione a pesar de las dificultades.

10.6. Cuando encuentre mi "amor verdadero", probablemente lo sabré poco después de encontrarnos.

10.7. Estoy seguro/a de que cada nuevo aspecto que conozca acerca de la persona elegida para un compromiso a largo plazo, me agradará.

10.8. La relación que tenga con mi "amor verdadero" será casi perfecta.

10.9. Si amo a alguien, encontraré la forma de que estemos juntos con independencia de la oposición que exista, distancia física, o cualquier otra barrera.

10.10. Habrá un solo amor verdadero para mí. 
10.11. Si la relación que mantengo es la que se supone tiene que ser, cualquier obstáculo (por ejemplo, falta de dinero, distancia física, conflictos en el trabajo) podrá superarse.

10.12. Si encuentro a la persona adecuada, es muy probable que me enamore casi de inmediato.

10.13. Espero que en mi relación de pareja, el amor romántico realmente dure; que no desaparezca con el tiempo.

10.14. La persona a quien ame será la pareja romántica perfecta; por ejemplo, él/ella será tolerante, comprensivo/a y me amará.

10.15. Creo que si mi pareja y yo nos queremos, podemos superar cualquier problema que nos surja.

11. Indica el nivel de acuerdo o desacuerdo con cada uno de los siguientes ítems (teniendo en cuenta que $1=$ Nada de acuerdo, 2 =Algo de acuerdo, 3 = Bastante acuerdo y $4=$ muy de acuerdo).

11.1. Por el bien de sus hijos, aunque la mujer tenga que soportar la violencia de su marido o compañero conviene que no le denuncie

11.2. Si una mujer es maltratada por su compañero y no le abandona será porque no le disgusta del todo esa situación

11.3. Cuando una mujer es agredida por su marido, algo habrá hecho ella para provocarlo

11.4. Un buen padre debe hacer saber al resto de su familia quién es el que manda

11.5. Para tener una buena relación es deseable que la mujer evite llevar la contraria al hombre

11.6. La violencia que se produce dentro de casa es un asunto de la familia y no debe salir de ahí

11.7. Está justificado que un hombre agreda a su mujer o a su novia cuando ella decide dejarle 